\title{
LA BAIGNOIRE, LE LIT ET LA PORTE. LA VIE SOCIALE DES OBJETS DE LA PSYCHIATRIE
}

\author{
Benoît Majerus \\ Belin | Genèses \\ $2011 / 1-n^{\circ} 82$ \\ pages 95 à 119
}

ISSN 1155-3219

Article disponible en ligne à l'adresse:

http://www.cairn.info/revue-geneses-2011-1-page-95.htm

Pour citer cet article :

Majerus Benoît , « La baignoire, le lit et la porte. La vie sociale des objets de la psychiatrie », Genèses, 2011/1 n 82, p. 95-119.

Distribution électronique Cairn.info pour Belin.

(c) Belin. Tous droits réservés pour tous pays.

La reproduction ou représentation de cet article, notamment par photocopie, n'est autorisée que dans les limites des conditions générales d'utilisation du site ou, le cas échéant, des conditions générales de la licence souscrite par votre établissement. Toute autre reproduction ou représentation, en tout ou partie, sous quelque forme et de quelque manière que ce soit, est interdite sauf accord préalable et écrit de l'éditeur, en dehors des cas prévus par la législation en vigueur en France. II est précisé que son stockage dans une base de données est également interdit. 


\section{La baignoire, le lit et la porte. \\ La vie sociale des objets de la psychiatrie*}

\section{Benoît Majerus}

PP. 95-119

Le 5 juillet 1938, le directeur de l'hôpital Brugmann rédige à l'intention de Georges Vellut ${ }^{1}$ ingénieur à la Commission d'assistance publique (CAP), une note relative à la construction de toilettes supplémentaires à l'Institut de psychiatrie $^{2}$ (voir document 1 ).

À la fin de cette lettre se trouve un paragraphe qui décrit les détails de la porte. Selon ces instructions, Vellut dessine un avant-projet qui permet la surveillance du reclus, notamment par une porte semi-transparente (voir document 2). Celle-ci cache à peine, dès lors, les utilisateurs des toilettes. Les cinquante centimètres d'ouverture en bas, prévus sur l'esquisse, permettent de voir une personne qui se tiendrait debout sur la cuvette des toilettes, haute de quarante centimètres. Et la hauteur de la porte, un mètre cinquante à compter du sol, permet à toute personne de taille moyenne de jeter un coup oil sur l'intérieur.

Les toilettes sont, pour un patient, l'unique endroit de l'Institut qui ne soit pas collectif. Le dortoir, la salle de bain, le parc... tous les autres «espaces psychiatriques» (Topp, Moran et Andrews 2007) sont des espaces partagés qui ne permettent pas d'échapper au regard des autres patients ou du personnel soignant et médical. Cette visibilité totale est un des principes théoriques des espaces d'enfermement qui se construisent au XIXe siècle et qui restent valables jusqu'à aujourd'hui (Foucault 1975). Il n'est donc guère étonnant qu'un des manuels de psychiatrie les plus lus par les infirmiers mette ses lecteurs en garde envers ce lieu:

«La surveillance des W.C. doit être aisée; des suicides ont eu lieu pendant les minutes où, pour ménager sa pudeur, on a laissé un malade y aller seul».

(Morgenthaler et Forel 1940 : 135)

À partir de la fin des années 1920, le directeur de l'Institut de psychiatrie, le psychiatre Guillaume Vermeylen, entretient avec l'ingénieur Georges Vellut un 


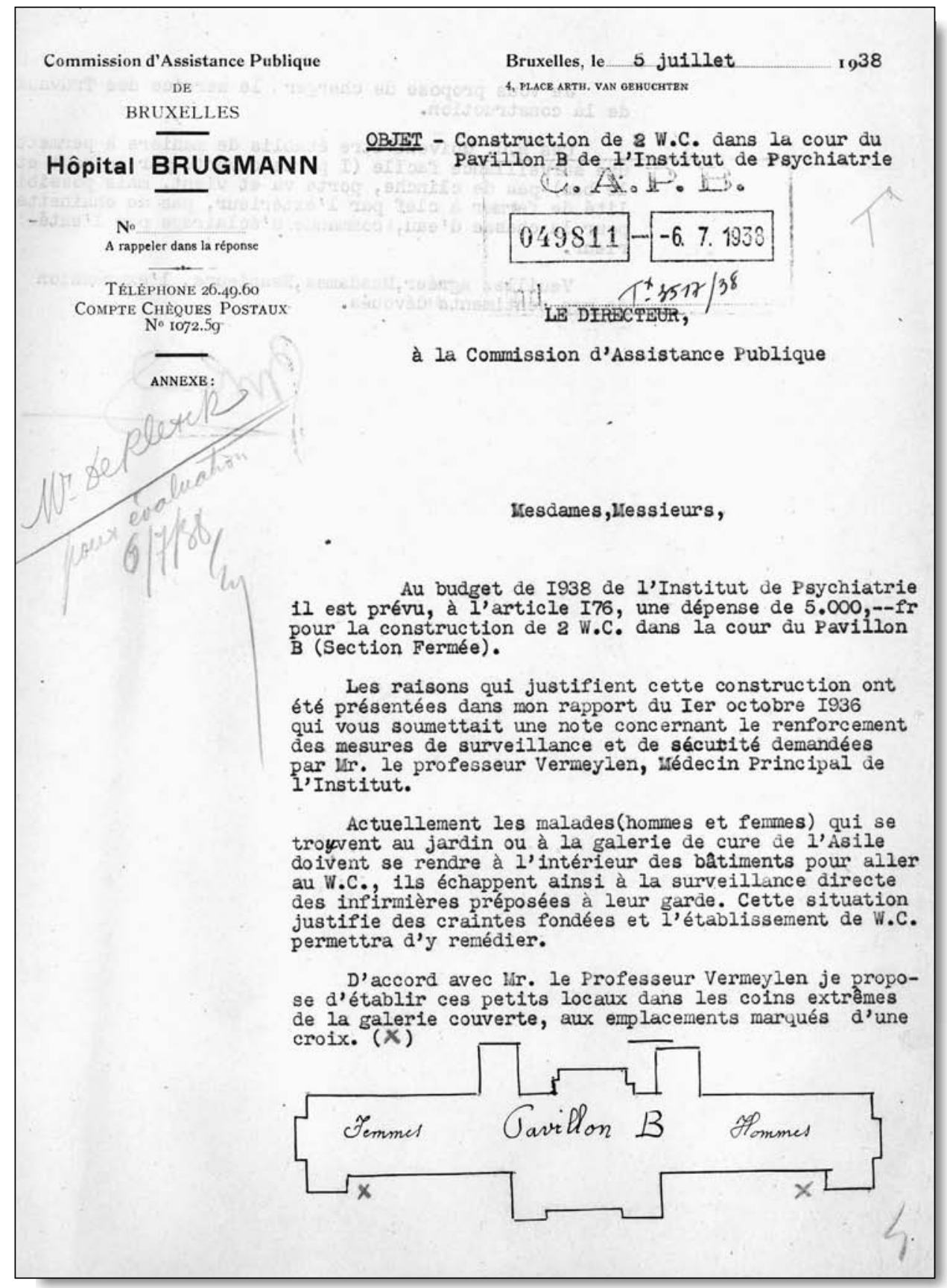

Document 1. Lettre du directeur de l'hôpital Brugmann à Georges Vellut. (C) Archives de la Commission publique d'action sociale de Bruxelles (ACPASB), travaux, boîte 219, lettre du directeur de Brugmann à la CAP (5 juillet 1938). 


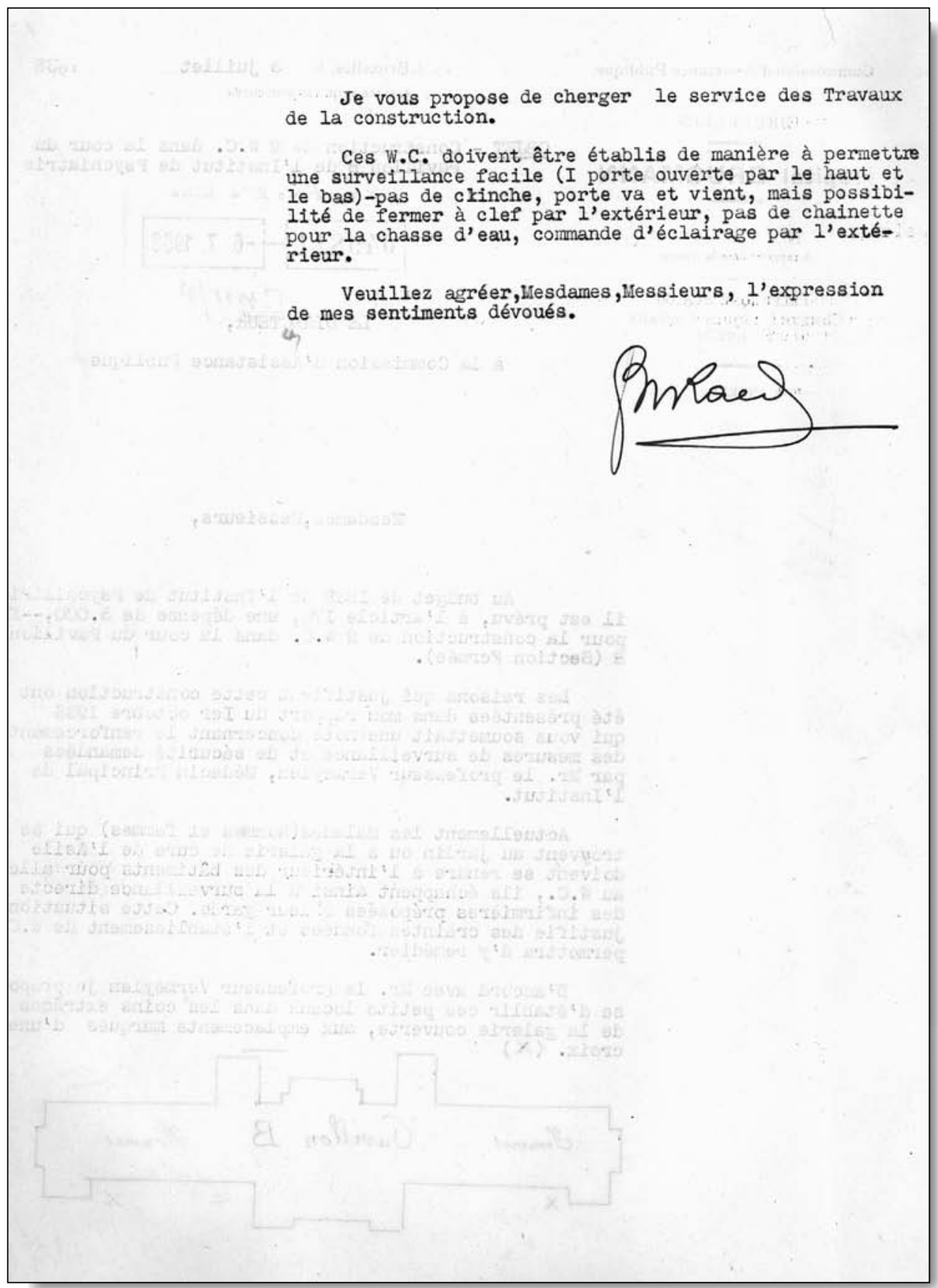

of

되

-

U

u

O

ค

1

$\infty$

of

0

드 


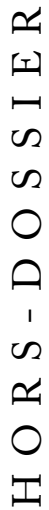

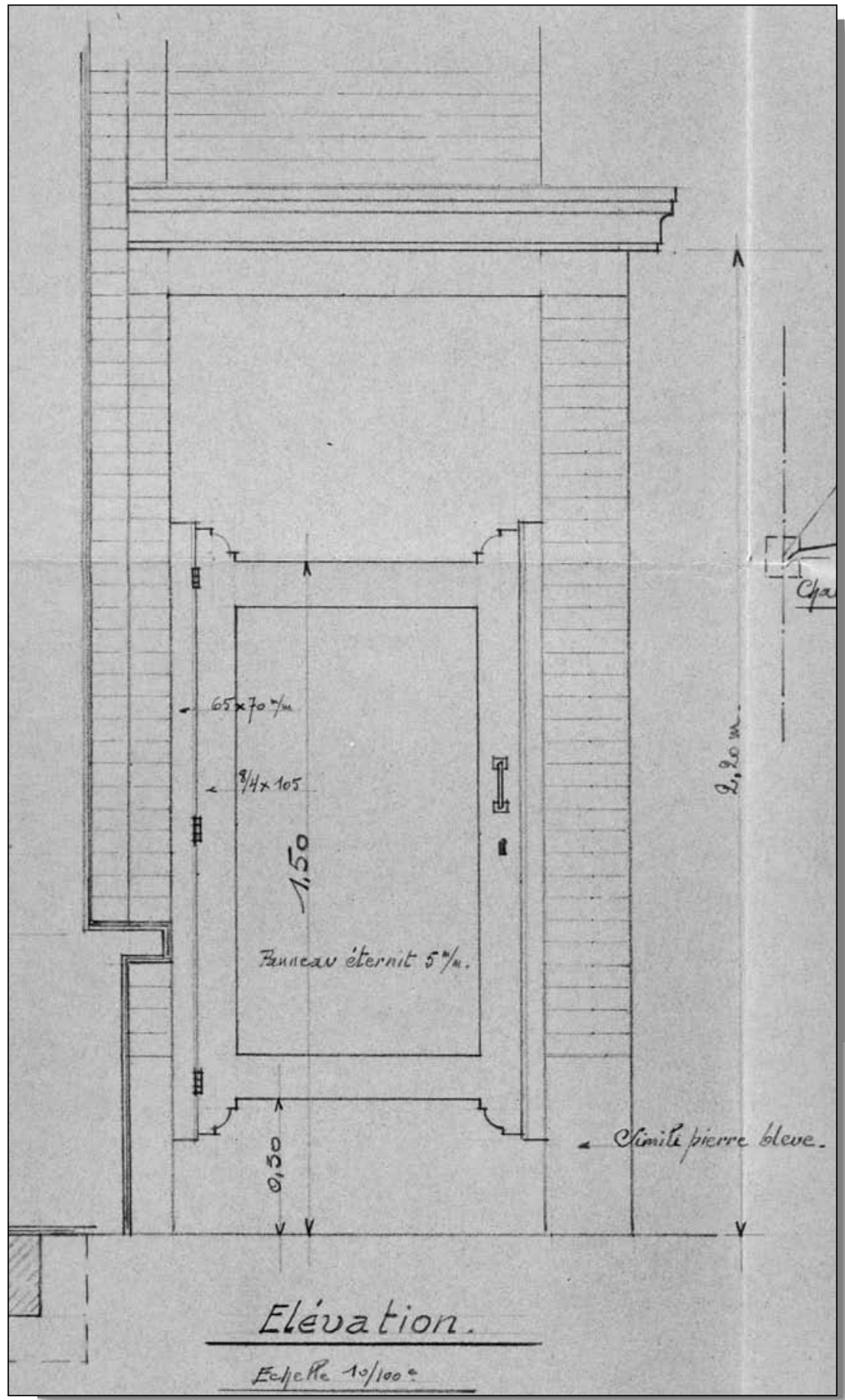

Document 2. Projet d'une porte de toilette. C ACPASB, travaux, boîte 30 . 
échange épistolaire soutenu. D'abord en vue de construire un hôpital psychiatrique - ce qui sera chose faite en 1931, et Vermeylen en sera le premier directeur - ensuite pour adapter le bâtiment aux problèmes nés de son utilisation. Cette correspondance a lieu aux lendemains d'une grande vague de construction asilaire en Europe et aux États-Unis, commencée au milieu du XIXe siècle et achevée pendant la Première Guerre mondiale. Indépendamment des choix architecturaux, ces asiles se caractérisent par leur forte capacité d'accueil et par leur construction à la marge des villes, voire à la campagne (Yanni 2007). En suivant les discussions de Vermeylen et de Vellut, le chercheur en sciences humaines découvre une véritable mine qui témoigne de la culture matérielle de la psychiatrie qui s'est progressivement constituée durant ces décennies: lettres, plans, photos, prospectus publicitaires nous parlent des objets, instruments, meubles et immeubles de la psychiatrie, de leur conception et de leurs usages.

Depuis les années 1980, les sciences humaines appréhendent les objets non plus seulement en tant qu'éléments passifs, mais aussi comme acteurs: les objets prennent dorénavant sens dans l'interaction avec des individus et des groupes ${ }^{3}$. On peut donc partir de l'idée que l'expérience de la psychiatrie est déterminée par la configuration qu'imposent ces objets. Que ce soit l'objet architectural comme l'asile, premier agent thérapeutique de cette spécialisation, l'objet identifié comme spécifique à la psychiatrie telle que la camisole ou des objets plus communs de la vie hospitalière comme le lit, tous structurent l'action des différentes populations psychiatriques. Médecin, infirmière, assistante sociale, patient vivent la psychiatrie à travers ces objets. Ces «non-humains", pour reprendre l'expression de Bruno Latour (1987), conditionnent beaucoup plus fortement la psychiatrie que ne le laisse apparaître une historiographie focalisée sur une analyse des écrits de psychiatres. Une réflexion sur la folie et sa gestion, si elle veut dépasser les apories antagonistes de controverses héritées des années 1970 et $1980^{4}$, peut-elle profiter donc de ce retour vers l'objet quotidien et son vécu? Cet angle permet de répondre à deux problèmes qui se posent dans une réflexion sur la psychiatrie en particulier, et sur la médecine en général. Le premier est l'écart entre discours théorique et mise en action pratique. Analyser l'objectivation du discours médical, la mise en objets des théories psychiatriques est une façon de relire ce questionnement (Attfield 2000 : 1). Ensuite, cela permet aussi d'observer l'appropriation de ces objets dans la pratique par des populations psychiatriques rarement impliquées dans «l'imagination» de l'objet, mais qui en sont les premiers utilisateurs, notamment les infirmières et les patients (Landzelius 2001). Il s'agit ici de traquer la manière dont infirmières, patients et objets font preuve de Eigensinn (Lüdtke 1994), terme que certains auteurs français ont joliment traduit par «subjectivité rebelle». À travers la baignoire - un outil évident du traitement - le lit - un meuble hospitalier banal - et la porte - artefact immobile passant le plus souvent inaperçu, la «vie sociale des objets» psychiatriques constituera le fil rouge de cet article. Le choix de ces trois objets aux 


\section{L'institut de psychiatrie à l'hôpital Brugmann}

Inauguré en 1931, I'Institut est assez singulier dans l'univers psychiatrique belge tel qu'il se structure dans le premier xxe siècle autour des grands asiles tenus par les congrégations religieuses. II est, d'abord, à la fois un lieu de formation pour les étudiants en médecine de l'Université libre de Bruxelles et un rouage de la Commission d'assistance publique de la municipalité de Bruxelles: fonctions universitaire et charitable y sont en tension. Les enseignants sont à la recherche de "cas médicalement intéressants" alors que beaucoup de patients s'y retrouvent pour des raisons d'exclusion sociale (alcoolisme, vieillesse...). Seconde particularité: I'Institut y est inscrit dans un complexe hospitalier/médical, celui de l'hôpital Brugmann. Ce dernier a été construit dans le style pavillonnaire au cours des années 1920. Cette intégration de la psychiatrie dans un hôpital est très rare à l'époque. Intégration relative puisque I'Institut se trouve d'ailleurs à la marge, à côté de la morgue, de ce grand ensemble hospitalier. L'hôpital Brugmann et l'Institut sont pensés dans un double mouvement qui peut paraitre contradictoire: s'inscrire dans la modernité tout en y voyant la cause profonde de la maladie (mentale). Installé à l'écart de l'agglomération bruxelloise, I'Institut se trouve dans "une ambiance atmosphérique aussi pure que possible», loin de l'agitation de la ville. En même temps, les responsables politiques et médicaux revendiquent la modernité du traitement qui se traduit notamment par la création d'une section ouverte et par une attention portée, au moins dans le discours, aux fondements neurologiques de la psychiatrie.

caractéristiques différentes répond à la volonté d'analyser des objets qui sont présents dans le quotidien et se caractérisent donc par une certaine banalité, mais qui en même temps ont pris des fonctions spécifiques dans l'espace psychiatrique au XXe siècle. Ils offrent donc l'avantage heuristique de dire la psychiatrie sans se réduire à ce champ médical.

\section{Baignoire}

À l'inauguration de l'Institut, l'hôpital édite un livret de cartes postales sur le nouveau bâtiment (voir document 3). Elles présentent une institution immaculée, sans patient, contrairement au livret édité auparavant lors de l'inauguration de l'hôpital lui-même. Dans cette série d'une dizaine d'illustrations, apparaît un seul objet spécifiquement lié à la thérapie psychiatrique, seul domaine médical où s'exerce sa vertu thérapeutique: la baignoire. La carte postale annonce pudiquement que celle-ci n'est pas destinée aux fins hygiéniques habituelles, mais à des «bains prolongés».

Les baignoires se trouvent dans une seule salle, sans aucune séparation entre elles. L'absence de toute intimité ressort ainsi nettement de cette photo, qui témoigne aussi de la mutation de la baignoire ordinaire dans un milieu psychiatrique. Contrairement aux baignoires installées pour les infirmières dans un bâtiment voisin, également bâti au début des années 1930, celles de l'Institut n'ont pas de robinet individuel: l'objet est potentiellement dangereux pour le patient, et peu fonctionnel par sa rigidité. Un tuyau, fixé au mur et inaccessible aux 


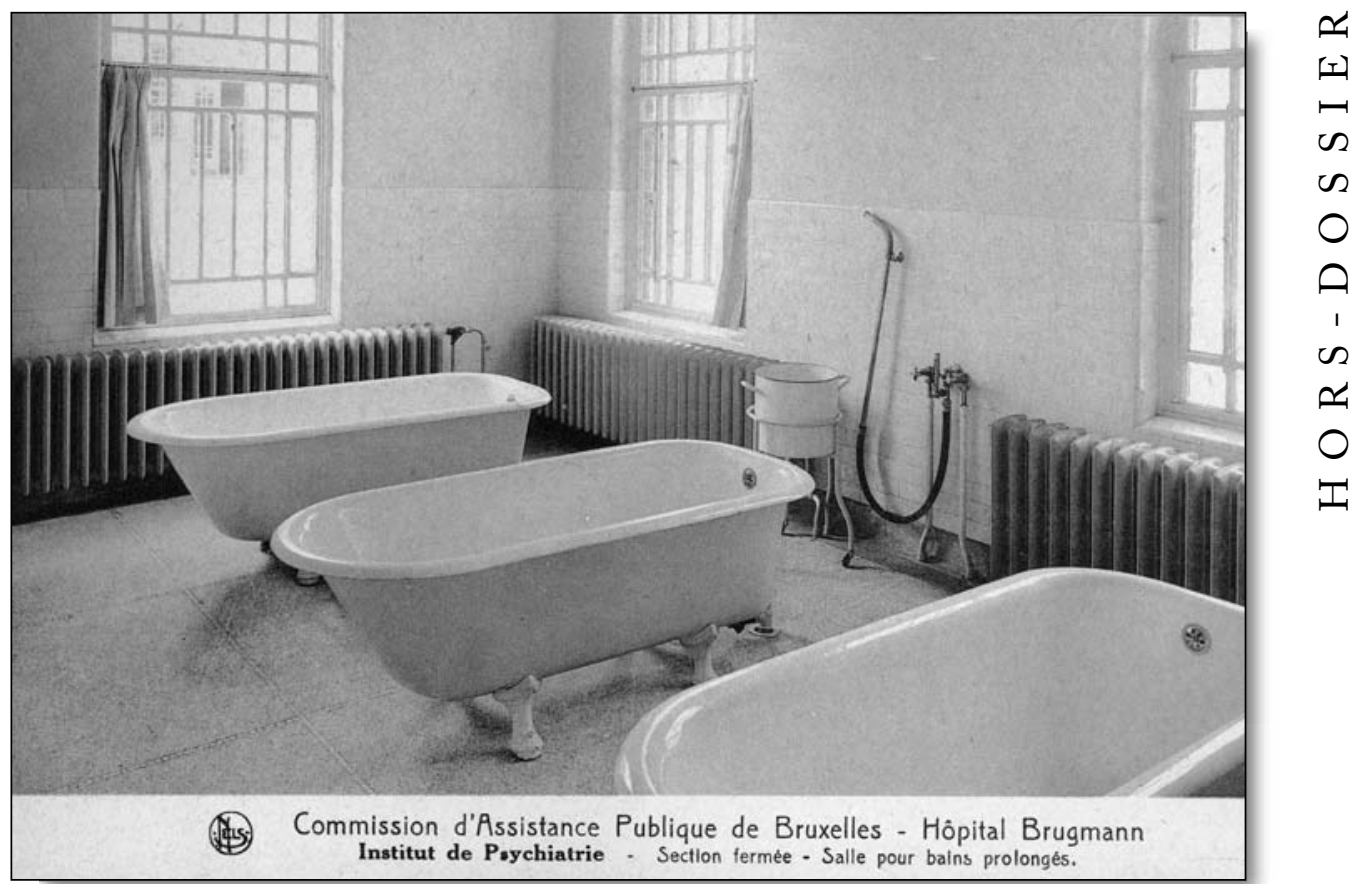

Document 3. Carte postale éditée en 1931 représentant les baignoires à l'Institut de psychiatrie. (C) ACPASB, fonds iconographique, H/H.B/196.

patients, donne une plus grande marge de manœuvre à l'infirmière. Malgré cette adaptation matérielle du dispositif, mettre le patient dans le bain reste un exercice périlleux, comme en témoignent les nombreux «accidents de travail» enregistrés dans cette pièce. D'ailleurs, la salle de bain en général et la baignoire en particulier montrent rapidement leurs limites dans la pratique quotidienne. L'objet imaginé et construit ne s'avère pas toujours fonctionnel. Ainsi des radiateurs, qui en 1931 ne sont pas encore protégés, se voient adjoindre des protections spécifiques après que deux patients se furent blessés sur leurs arêtes ${ }^{5}$. Il en va de même concernant le dispositif d'enfermement, une planche spéciale boulonnée sur la baignoire et percée d'une ouverture circulaire où le malade ne peut que passer la tête. Attribut de la baignoire psychiatrique, et non représenté sur la carte postale, il est vite jugé «rébarbatif» par le directeur de l'Institut qui demande à l'ingénieur de concevoir des fermetures en caoutchouc ${ }^{6}$. Dernier élément qui pose rapidement problème: l'eau. Amenée par tuyaux souterrains depuis l'hôpital Brugmann, elle est rarement à la température optimale de $36^{\circ}$ lorsqu'elle arrive à l'Institut. Le dispositif du bain est d'ailleurs tout entier remis en cause, car la durée des bains flétrit la peau de telle manière que les patients ne sont parfois plus capables d'utiliser leurs mains ou de marcher. Pour y remédier, le personnel bricole un nouveau dispositif, le «bain sec, consistant en l'aménagement 
d'une espèce de matelas formé de coussins et d'une couverture sur le malade avant de recouvrir le tout d'une planche de bain chez les hommes ou d'une bâche chez les femmes» ${ }^{7}$. Ce «bain sec» diminue la charge de travail des infirmières, qui ne doivent pas contrôler la température de l'eau ni se soucier de l'état de la peau du patient. C'est là un des indices de la réinvention de la baignoire par la pratique et la théorie psychiatrique, tant vis-à-vis de son utilisation commune qu'au regard de son usage ordinaire en milieu hospitalier.

En effet, au début du XXe siècle, l'hôpital s'était inscrit dans le grand récit de l'éducation hygiénique des masses (ouvrières) à côté de l'école ou de l'armée. Antoine Depage, l'un des médecins belges les plus influents du moment, l'affirmait sans ambiguités dans son ouvrage consacré à la «construction des hôpitaux» :

«On devrait profiter du passage de l'ouvrier à l'hôpital pour l'initier aux règles de l'hygiène privée. On rendrait un immense service aux pensionnaires de l'hôpital en leur apprenant à se laver les dents et à se curer les ongles. L'hôpital pourrait, aussi bien que l'école, contribuer à l'éducation populaire [...] il est superflu d'insister sur les inconvénients qu'il y a à contraindre quelqu'un à faire ses ablutions en public, dans la salle de malades»

(Depage, Vandervelde et Cheval 1912 : 174).

Les «bains de propreté» sont également fortement préconisés en asiles «au moins une fois par semaine» (Mignot et Marchand 1931 : 115). Parallèlement à ces considérations d'hygiène, la baignoire remplit trois fonctions particulières à l'asile psychiatrique: juger le degré d'acceptation de l'internement, retracer l'histoire du patient à travers la lecture du corps, et assurer une mission thérapeutique.

D'une part, le passage par la baignoire constitue une étape centrale dans le «processus de mortification» décrit par Erving Goffman. Chaque reclus - que ce soit en psychiatrie, en prison ou au couvent - est dépouillé des éléments lui permettant de maintenir la «représentation de lui-même» (Goffman 1968 : 56) avant son enfermement. La transformation de l'identité passe toujours par les mêmes étapes: mise à nu, enlèvement de tous les éléments personnels, exposition au regard de l'autre, qui signifient sans mot dire l'asymétrie de pouvoir qui régit l'intérieur de l'institution. La baignoire constitue un dispositif central dans ce processus de mortification. Si le bain ne se déroule pas dans le dortoir, le faire couler est un signe pour les autres patients qu'il y a un nouvel entrant et provoque une certaine agitation dans les dortoirs. L'entrée dans la baignoire constitue souvent la première contrainte à l'intérieur de l'Institut, et peut déclencher une spirale d'enfermement et de violence. Margot R., une jeune femme de seize ans, est internée en novembre 1956 pour «maniérisme, agitation». Le dossier ne permet pas de reconstruire exactement son parcours avant l'internement à l'Institut, mais laisse supposer que son environnement familial estime qu'elle a un comportement qui ne sied pas à son âge: "elle boit d'importantes quantités d'alcool» et entretient des «relations avec des Italiens qui sont locataires chez ses 
parents ${ }^{8}$. Cette jeune fille, en rupture avec son milieu familial, n'accepte guère le passage au bain qui signifie son entrée dans l'institution psychiatrique:

«Malade entrée vers 17 heures excessivement agitée. Crie se débat, crie fort ses cris ne parle pas mise à la baignoire avec difficultés quelque temps après il est encore impossible de la sortir. Au bain dort dès qu'on la touche s'agite à nouveau et se débat (pouls est bon).

Soir: calme dans la baignoire, dort. A été mise sous toile9, n’a presque pas bougé, s'est rendormie aussitôt ${ }^{10}$ ».

Dans le cas de Margot R., le bain, initialement instrument hygiénique et d'observation, se transforme tout de suite en mesure de contrainte. La décision de placer quelqu'un dans la baignoire est le plus souvent prise par les seules infirmières qui gèrent le quotidien dans une salle. Le bain dure régulièrement plus de six heures, se poursuivant parfois sur plusieurs jours. Ainsi en 1939, Valentine M. est transférée du secteur de la médecine interne vers l'Institut. Âgée de trentequatre ans, cette femme restera pendant trente-cinq jours en psychiatrie. La patiente, après avoir «ri sans raison et [...] jeté son gobelet par la fenêtre», est mise au bain dès son entrée. Comme elle reste agitée, elle passe vingt-six heures dans la baignoire, avec seulement deux interruptions de quinze minutes ${ }^{11}$.

D'autre part, deuxième «raison» du passage par la baignoire: lire le corps du patient. En psychiatrie, la parole du patient est par principe douteuse. Les traces $\mathrm{du} /$ sur le corps sont a contrario considérées comme véritables. La lecture du corps nu est un élément important du protocole d'entrée, qui a laissé sa trace dans les fiches des infirmières des dossiers de patients. Ainsi celui d'Alex F., le premier jour: «La malade présente de nombreuses ecchymoses sur les bras et les jambes ${ }^{12}$ ». De même, dans le dossier d'Emmanuelle R., une ménagère de cinquante et un ans internée pour «hypochondrie» en 1960: «A un très grand bleu sous son bras droit et une petite blessure à sa main droite ${ }^{13}$ ».

Ces signes, parfois, permettent de reconstruire un fragment de l'histoire (psychiatrique) du patient. En mai 1940, Catherine M., âgée de vingt-cinq ans, entre pour la deuxième fois à l'Institut. Les notes dépeignent une jeune femme très anxieuse. Lors de cette deuxième entrée, l'infirmière note: "Relativement propre de linge et de corps. Présente au poignet et dans le creux du cou des anciennes cicatrices de T.S. [tentative de suicide] ${ }^{14}$ ». Dans les fiches, ces stigmates sont souvent relevés avec grand soin, mais rarement interprétés. À partir de 1936, Charline H. est hospitalisée cinq fois en quatre ans. À chaque séjour, l'infirmière constate des ecchymoses. Lors du dernier passage en 1940, les notes laissent supposer que la patiente est soumise à ce qu'on appellerait aujourd'hui des violences conjugales:

"présente plusieurs ecchymoses sur tout le corps principalement aux bras et aux jambes quelques égratignures? au cou; raconte qu'elle a déjà passé un séjour ici, dit qu'elle ne sait plus tenir son ménage alors se met à crier et que son mari se fâche ${ }^{15}$ ». 
Dans une écriture extrêmement euphémisante, les signes de la violence que révèle ici la lecture précise du corps sont dissociés de l'acte même ayant conduit à ces «bleus». L'anthropologue américain Robert Barrett a souligné à ce sujet la différence d'approche entre l'infirmière et le médecin. Ce dernier, par les examens physiques s'intéresse surtout à la physiologie interne, alors que les infirmières travaillent essentiellement «la surface du corps» (Barrett 1996 : 54). À ce titre, la baignoire est leur outil d'examen clinique, l'analogue de ces dispositifs de lecture de l'intérieur des corps qui vont se multiplier depuis les années 1950 en psychiatrie: l'électroencéphalogramme, l'électrocardiogramme ou la radioscopie (Borck 2005).

Enfin, troisième rôle particulier de la baignoire dans l'asile: sa fonction thérapeutique. Les bains chauds sont en effet longtemps l'unique moyen dont le personnel dispose pour calmer un patient. La méthode n'est pas neuve: c'est au moins depuis le milieu du XIXe siècle qu'ils sont régulièrement utilisés comme thérapie psychiatrique (Braslow 1997 : 11-12). En 1905, lors du 15e congrès des médecins aliénistes à Rennes, un rapport est exclusivement consacré à la question, et la balnéothérapie reste jusqu'à la fin des années 1920 «un des éléments principaux dans la thérapeutique psychiatrique» (Régis 1923 : 1007). Les explications sur sa fonction guérisseuse sont multiples et rarement concordantes - élimination des éléments toxiques par la peau, amélioration de la fonction cérébrale par l'influence sur le système vasculaire, etc. Le manuel de Morgenthaler et Forel, déjà cité, avance la théorie suivante: "L'eau est un milieu plus dense, offrant par conséquent plus de résistance que l'air; de ce fait, elle calme les malades» (1940: 137). Quoi qu'il en soit, pour les psychiatres, la baignoire n'est pas uniquement pensée comme un moyen de rétention mais s'inscrit clairement dans un contexte thérapeutique.

En face des premières thérapies biologiques (malariathérapie, cure d'insuline...) qui font leur apparition dans les années 1930, le bain est pourtant une thérapie peu spectaculaire, jugée peu «scientifique». En 1936, le journal satirique bruxellois Le Pétard se gausse du directeur de l'Institut: «la baignoire [...] c'est tout ce que Monsieur le Professeur Vermeylen qui enseigne à l'Université de Bruxelles a trouvé de mieux pour calmer des malades nerveux ${ }^{16} »$. Les bains, par ailleurs, n'apaisent pas nécessairement, comme en témoigne une lettre de James B., représentant de commerce de trente-huit ans atteint de paralysie générale:

«Je suis donc pour le deuxième jour en bas, je m'y plais beaucoup [...] Je dors mieux qu'en haut, car au 48 il y a des hommes dans le bain qui hurlent toute la nuit ${ }^{17}$ ».

Le côté «bricolage» ressort d'un autre écrit de James B. Dans une première lettre à sa femme, il assimile à la fois le bain à un outil d'enfermement mais également à un moyen d'humiliation:

«Voilà donc que je suis à Brugmann je dois te dire que contrairement à la dernière fois je suis très bien, bonne nourriture mes ils m'ont fait une mauvaise blague ils 
m'ont mis dans un bain complètement recouvert d'une planche dont la tête seule dépassait, je suis resté la dedans depuis hier $5 \mathrm{~h}$ jusqu'à ce matin onze heure, je leur ai demandé de sortir pour faire ma grande, ils ont refusés disant de faire dans le bain, je suis donc resté deux heures dans cette infusion ${ }^{18}$ ».

À l'Institut, finalement, le recours à la balnéothérapie ne se décline guère en fonction d'un diagnostic, mais essentiellement en fonction de l'état d'agitation du patient. Liée à la procédure d'entrée en psychiatrie, elle touche environ $10 \%$ des malades dans les années 1930 et 1940. À la fin des années 1940, le bain continue à être utilisé, mais à de plus rares occasions. Pour certains patients, la baignoire devient même refuge. Entre octobre 1953 et octobre 1956, Michel M. va effectuer six séjours à l'Institut, séjours entrecoupés par des passages dans d'autres institutions psychiatriques. Considéré la première fois comme "maniéré »19, Michel M., diagnostiqué "psychasthénique», commence lors de son cinquième séjour à se réfugier de plus en plus dans la salle des baignoires. Une fois sorti du lit le matin, il cherche la solitude par des bains prolongés.

«Est resté toute l'après-midi dans la salle de bain. Malgré qu'on enlève le bouchon et l'eau de la baignoire, y reste couché sans eau. Ne veut pas se laisser raisonner, se débat avec force quand on veut le faire sortir ${ }^{20}$ ».

Le bain devient ainsi un moment de calme et de solitude. À compter des années 1960, la baignoire disparaît des archives du soin, et son usage se restreint à sa fonction hygiénique 21 .

\section{Lit}

Au fil du XVIII' siècle, le lit est devenu un élément caractéristique de l'hôpital. Dans la deuxième moitié du XIXe siècle, les aliénistes, dans leur effort pour intégrer le champ médical, vont organiser les salles des asiles comme dans les hôpitaux généralistes: autour des lits (Boschma 2003 : 65-67). Élément de mesure qualitative, le lit est aussi une unité quantitative. Il sert à exprimer la dimension d'un hôpital. Ainsi, lors des discussions sur la taille de l'Institut, les responsables politiques et médicaux parlent d'un hôpital "de 116 lits», et non pas "pour 116 patients». Les lits ont des "prix journaliers», des "prix annuels», ce qui permet de comparer le coût des différents services, ou encore de calculer la surpopulation, ou la sous-population. La réforme des hôpitaux psychiatriques à partir des années 1960 se fait, entre autres, sous le mot d'ordre de la «réduction des lits psychiatriques». Mais le lit est aussi un objet qui se plie aux conceptions et aux usage propres à l'Institut (voir document 4).

À première vue, le lit dessiné par Vellut est semblable à ceux des autres services de l'hôpital Brugmann (voir document 5). 


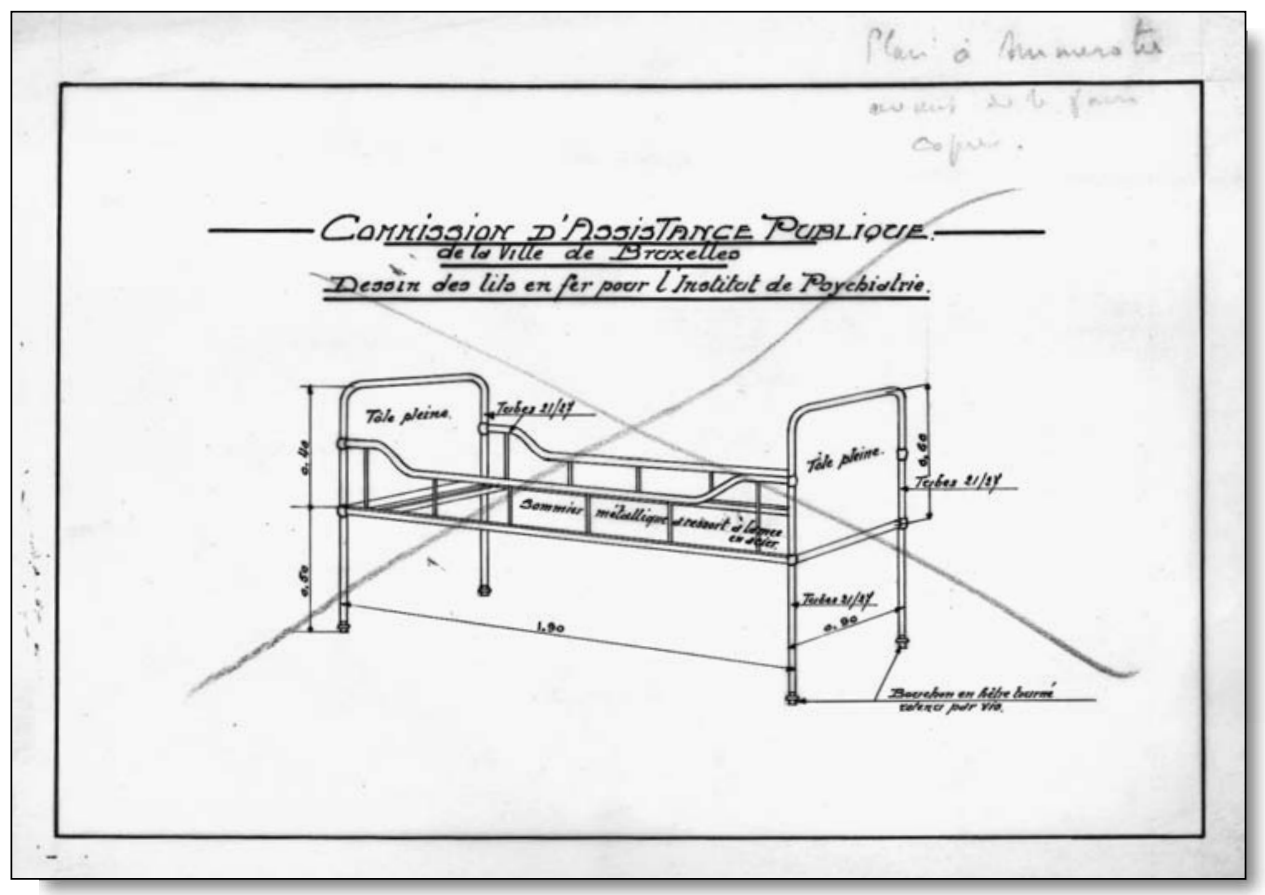

Document 4. Projet d'un lit de psychiatrie, dessiné par Georges Vellut. @ ACPASB, travaux, boîte 30 .

Construit en fer avec des angles arrondis, ce lit est immédiatement identifiable comme élément d'un mobilier hospitalier. De nombreux détails de dessin font néanmoins apparaître la spécificité psychiatrique. À l'Institut, cette différence s'exprime d'autant plus clairement que le bâtiment s'inscrit dans un dispositif hospitalier plus large - celui de l'hôpital Brugmann. La direction de l'hôpital se livre en permanence à une comparaison entre les services "normaux» et l'asile, comparaison qui aboutit à une différenciation - et non une assimilation entre psychiatrie et médecine. En effet, le directeur de l'hôpital souligne à plusieurs reprises qu'il faut tenir compte de la spécificité psychiatrique dans la matérialité des objets quotidiens - lits, tables de nuit, étagères.

Chargé de construire ce lit idoine, Georges Vellut s'adresse dans un premier temps au ministère de la Justice afin de connaître la pratique dominante dans les autres institutions; le ministère, tutelle des asiles psychiatriques, conseille des lits fixés au sol car «l'institut de psychiatrie [...] recevra des malades de tout acabit: des agités, des violents, des suicidaires ${ }^{22}$. Le ministère suit en cela l'avis de la plupart des manuels de psychiatrie: «Les lits sont fixés au sol. Cette disposition est rendue nécessaire par les accès de violence des malades au cours desquels ils pourraient renverser le lit» (Mignot et Marchand 1931 : 306). Pourtant, c'est précisément cette immobilité qui fait finalement préférer une autre solution à 


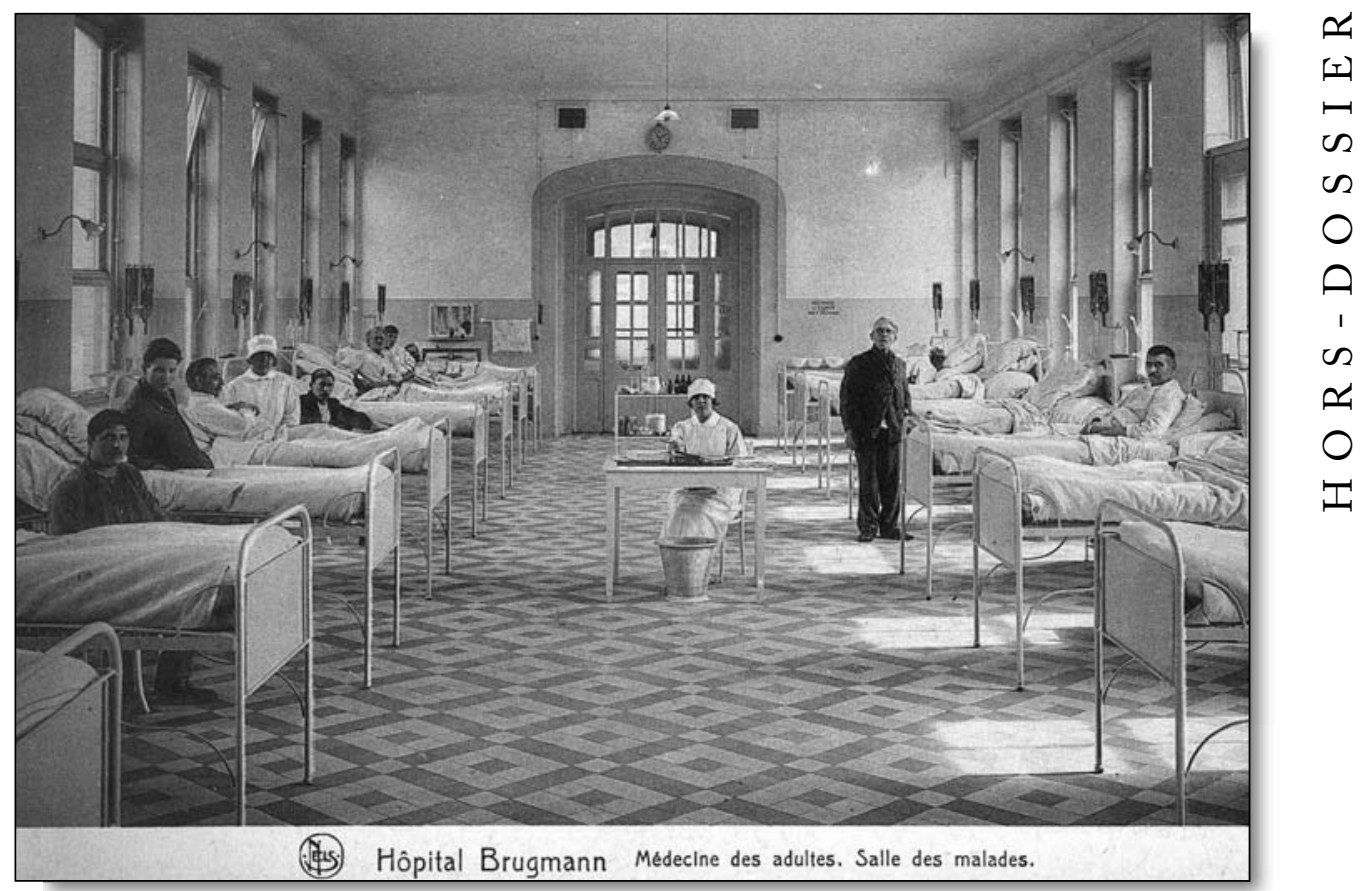

Document 5: Carte postale éditée en 1922 représentant une salle de l'hôpital Brugmann. (c) ACPASB, fonds iconographique, H/H.B/56.

Vellut, sur la base de ses contacts avec l'asile de Lovenjoel ${ }^{23}$. La mère révérende de Lovenjoel, plus concernée par la gestion quotidienne d'un asile que par des réflexions théoriques sur l'objet psychiatrique, conseille le lit mobile, qui permet d'utiliser l'espace de manière optimale en temps de surpopulation et qui facilite grandement le nettoyage des sols. L'Institut aura donc des lits mobiles. Néanmoins, pour obtenir une certaine stabilité, Vellut reprend une astuce proposée par la mère révérende: ce seront des lits «avec pieds en bois tourné au lieu de roulettes ce qui donne une fixité relative» 24 . Par ailleurs, et contrairement aux autres lits utilisés à Brugmann, ceux de l'Institut n'ont pas de barres à la tête et aux pieds, mais des panneaux en toile pleine afin d'éviter les tentatives de suicide à l'aide de draps attachés aux barres ${ }^{25}$. Ces lits sont construits de sorte qu'on puisse y attacher des planches pour empêcher les patients d'en sortir ${ }^{26}$. Le cahier des charges pour ces planches est très précis:

«Fourniture de 24 planches de lit en bois Canada 1er choix bien sec, épaisseur 4/4 de $1,80 \mathrm{~m}$ de longueur et $0,50 \mathrm{~m}$ de largeur suivant modèle existant [...] Ces planches seront pourvues de 2 fers plats (côté intérieur) de $30^{*} 6 \mathrm{~m} / \mathrm{m}$ et de 2 fers $U$ de $40 * 20 * 5$ extérieurement, maintenus par 3 boulons. Les fers auront une longueur de $65 \mathrm{~cm}, 40 \mathrm{~cm}$ sur les planches et $25 \mathrm{~cm}$ en-dessous avec trous et boulons à vis papillon formant charnière; en dessous partie plate de $5 \mathrm{~cm}$ de hauteur pratiquée 
aux fers U. Les planches seront renforcées par deux clamps en bois de chêne de 65 $\mathrm{m} / \mathrm{m}$ sur $4 / 4$ avec queue d'aronde entaillé à demi épaisseur des planches (côtés extérieurs). Les coins et toutes les arêtes seront arrondis. Il y aura deux trous dans la partie supérieure servant de poignées 27 ».

Les lits en psychiatrie ne sont pas équipés d'une petite lumière comme dans les autres services, pour éviter brûlures, coupures et électrocution. Si chaque reclus dispose d'un lit accompagné d'une table de lit, il n'a pas non plus de garde-robe propre, contrairement aux autres patients de l'hôpital Brugmann. Les vêtements des patients en psychiatrie sont conservés dans des armoires individuelles dans le sous-sol du pavillon $A$ auquel les patients n'ont pas accès 28 . Enfin, si le lit est jusqu'aux années 1950 le théâtre où la personne est transformée par l'écrit en patient, par l'écriture du dossier médical (Nolte 2009 : 35), les fiches des patients de l'Institut ne sont pas accrochées à leur lit. En psychiatrie, elles sont conservées dans la salle des infirmières, salle pour laquelle l'ingénieur fait construire une armoire à dossiers spécifique. Alors que dans les autres services le patient peut, s'il le désire, lire au moins une partie de son dossier médical, la chose n'est pas possible pour les patients psychiatriques.

Jusqu'aux années 1960, les patients sont systématiquement mis au lit à leur arrivée. Ce dispositif remplit plusieurs fonctions. Le lit est d'abord un symbole de

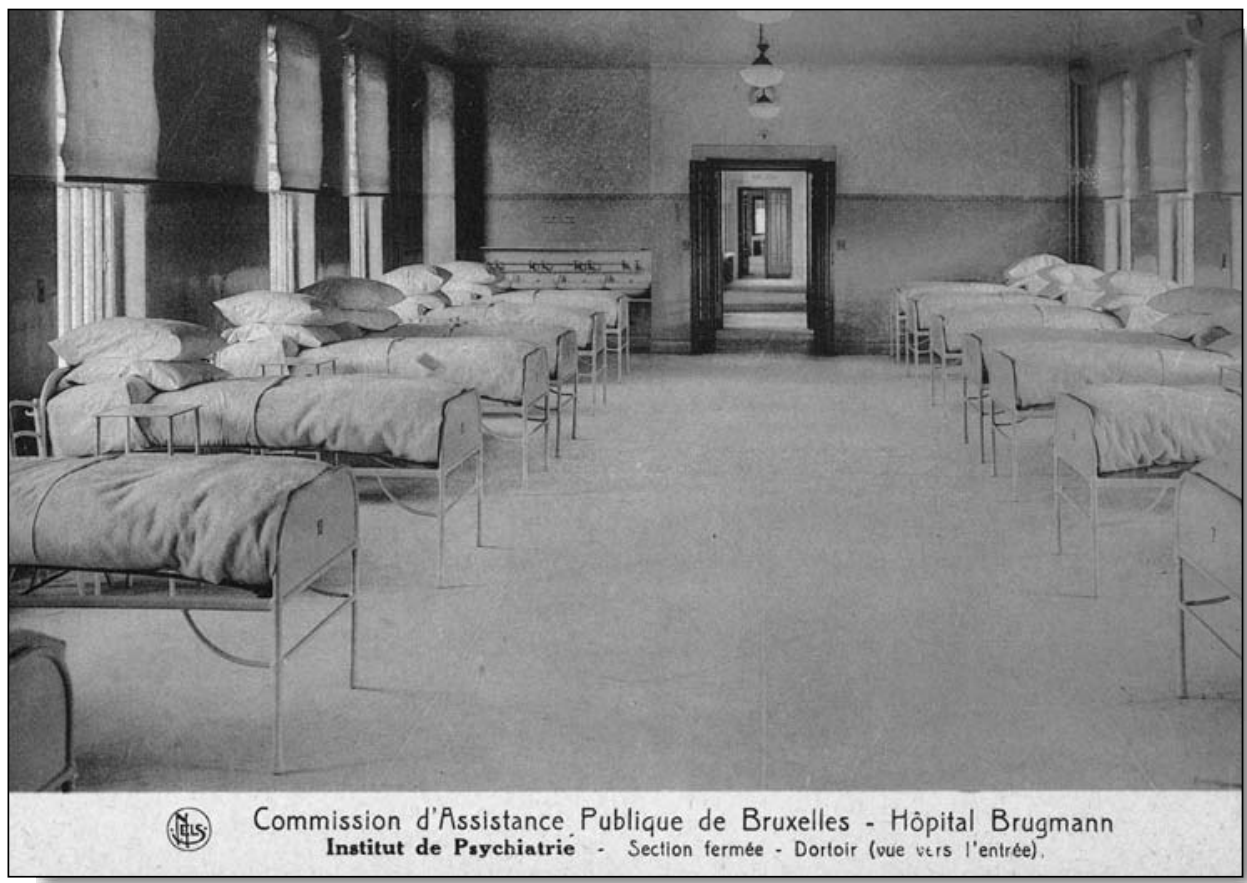

Document 6. Carte postale de la salle des agités de l'Institut de Psychiatrie (1931).

(C) ACPASB, fonds iconographique, H/H.B/196. 
l'hôpital en général: une personne alitée pendant la journée est souvent associée à une personne malade. Le lit devient symbole de la maladie, et son utilisation signifie donc également que la folie devrait être comprise dans le régime d'interprétation de la maladie et pas dans celui de la déviance sociale. Il constitue également un moyen de discipliner le corps et l'espace, à un moment où la plupart des malades ne se trouvent pas dans des chambres individuelles, mais dans des grandes salles communes. À l'Institut, celles-ci accueillent théoriquement dix-huit patients.

De même qu'une autre carte postale de la série, celle-ci nous montre une salle commune idéale - d'ailleurs dépourvue de patients. Les lits y sont disposés perpendiculairement le long des deux murs, créant ainsi un large couloir facilitant la circulation rapide à travers les salles. Entre chaque lit se trouve la distance nécessaire pour permettre la visite du médecin, mais également pour empêcher les patients de se toucher (voir document 6).

L'organisation spatiale de chaque salle est rythmée par l'agencement des lits, qui donne le ton de l'ordre et de la propreté. Ceci est vrai pour la médecine en général - il suffit de regarder les nombreuses photos de services médicaux où l'on voit les lits méticuleusement mis en rang - mais davantage encore en psychiatrie où le lit est l'outil central du contrôle du patient. Dans leur manuel de psychiatre, les deux médecins Mignot et Marchand soulignent d'ailleurs:

«Les lits doivent être très écartés les uns des autres afin que les malades ne puissent atteindre avec la main les sujets des lits voisins; ils doivent être éloignés des murs; il faut que les infirmiers puissent en faire facilement le tour pour maintenir les agités dans les moments d'excitation».

(1931: 307)

À l'intérieur de cet agencement, et dans chaque salle, les patients sont ordonnés selon la logique des lits qui remplissent ainsi une ultime fonction classificatoire: à l'Institut, celle-ci se décline d'abord d'après le genre - les hommes à droite, les femmes à gauche du pavillon - puis d'après l'agitation. Dans cet agencement, impossibilité pour le patient de devenir lui-même auteur de cet espace ou de se l'approprier directement (Parrott 2005). Cet ordre imaginé se heurte pourtant régulièrement à une réalité tout autre. Ainsi, à partir des années 1950, l'Institut est confronté de manière permanente à une surpopulation importante. Les règles énoncées par les manuels de psychiatrie (distance entre les lits) ne peuvent plus être respectées. L'organisation de la salle qui devait contribuer à combattre le désordre mental des reclus est déréglée. Une image extraite d'un documentaire montre l'encombrement des salles de l'Institut, du fait de la surpopulation$^{29}$ (voir document 7).

Les lits y apparaissent très rapprochés, au point que le grand couloir au milieu de salle est presque impraticable. Malgré le maintien d'une certaine symétrie, la salle donne une impression de tension. Le lit imaginé comme objet établissant l'ordre et permettant la circulation fluide du personnel devient objet encombrant. 


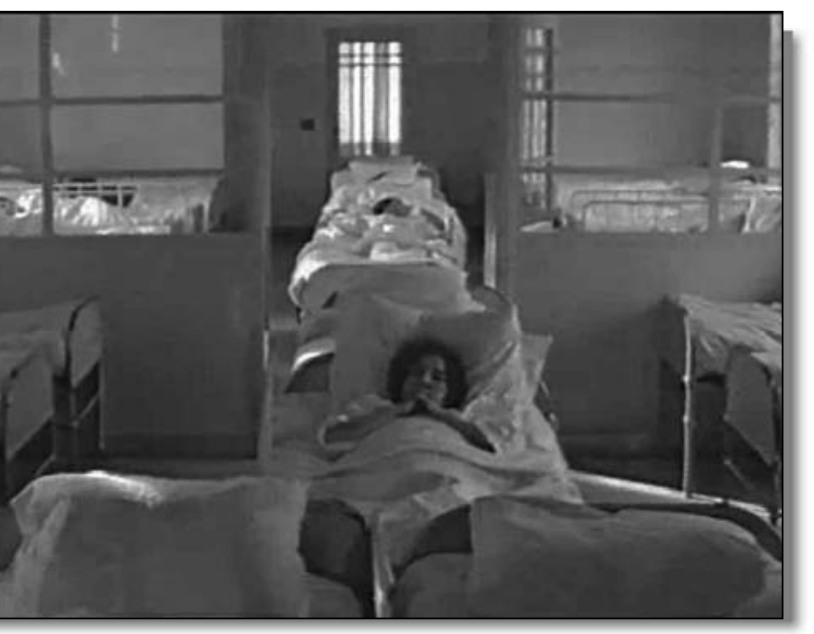

Document 7. Photogramme d'un reportage produit en 1964 sur la psychiatrie en Belgique. C RTB

Pour de nombreux psychiatres, le lit a valeur thérapeutique. Le repos du corps allongé conduit au repos des nerfs. L'excitation, produit de la vie urbaine moderne, est considérée comme l'une des premières explications du nombre croissant d'aliénés. À côté de cet argumentaire physiologique, le lit a également une fonction psychologique: reproduire le schéma en cours dans un hôpital «ordinaire» et ainsi convaincre la personne qu'elle est malade, et la pousser à reconnaître à l'asile et au psychiatre qui y travaille la même légitimité qu'aux autres médecins.

$\mathrm{Du}$ côté des infirmières, accepter d'être alité en plein jour est un indicateur quant au degré d'obéissance du nouveau venu et à son degré «d'autocritique», terme très usité à compter des années 1950 pour décrire la propension du patient à se dire malade. Gian Maria T. s'y conforme sans problème: «il se met calmement au lit» et la nuit se passe très bien, vu qu'il a «assez bien dormi»30. Plus souvent, cette étape pose problème. C'est là que le patient est concrètement confronté aux contraintes de l'institution. Bruno B. arrive en février 1933 à l'Institut pour un problème d'éthylisme. Il reste pendant vingt-deux jours en section fermée, avant de retourner dans sa famille:

«Est entré à 11 heures était calme et mis au lit. Fâché parce qu’il doit être au lit. Après plus agité. Crié toute force est mis au bain vers $31 / 2$ hres, reçu purge. Bain donné par Mlle M.31».

Dans ce cas, et ce n'est pas le seul, la mise au lit en pleine journée est considérée comme vexatoire et conduit le patient à se révolter contre le règlement en vigueur. Dès le premier jour, le patient fait alors l'expérience des limites de sa liberté et des moyens de coercition dont dispose le personnel soignant. Dans le cas de Bruno B., les conséquences sont assez lourdes: il ne sort des bains que quarante-huit heures plus tard, et est attaché au lit pour toute la journée.

Si le patient est particulièrement agité, il est tout de suite mis sous toile. Quelques-uns sont également soumis à cette mesure car jugés potentiellement dangereux. Ainsi Japhet A., de nationalité rwandaise, est amené à l'Institut en mars 1978 par la police: se considérant comme Dieu, il a refusé de payer son taxi. Malgré le fait qu'il soit calme lorsqu'il entre en salle - «traitement choc à la garde $\rightarrow$ somnolent ++ » - il est «mis préventivement sous bâche»32. La mise au 
lit obligatoire est en fait limitée aux sections fermées; dans les sections ouvertes, les patients peuvent dès la première journée circuler librement dans les dortoirs et nouer contact avec les autres hospitalisés.

Le lit est donc clairement pensé comme un «dispositif de l'institution totale» (Laé 2009), un outil disciplinaire. Mais les réformes et (apparentes) ruptures thérapeutiques qui affectent la psychiatrie dès les années 1950 et 1960 modifient aussi le rôle du lit ${ }^{33}$. L'hôpital psychiatrique se donne à présent comme fonction de favoriser la réintégration des patients dans la société, et on tente d'y simuler les conditions de vie et de travail du monde extérieur. Rester au lit pendant la journée n'est plus souhaité. Certes, les lits continuent à ordonner l'espace par leur disposition dans les services mais le personnel soignant et médical ne pratiquent plus de manière systématique l'immobilisation des patients agités pendant la journée. Rester au lit - (non)activité qui ne posait pas problème dans l'entre-deux-guerres devient un symptôme inquiétant à partir des années 1960 où des activités comme l'ergothérapie font leur entrée à l'intérieur de l'Institut.

C'est seulement alors qu'une autre réalité devient visible à travers les notes des médecins et les cahiers des infirmières: le lit comme premier espace privé dont dispose le reclus. Ce lit est souvent le seul espace qui est dévolu spécifiquement à un individu. Certes, tous les lits se ressemblent et il est strictement interdit de personnaliser l'objet au moins jusqu'aux années 1950. Même au milieu des années 1960, le photogramme du film ci-dessus (document 7) montre que les lits ne constituent pas encore des espaces personnalisés. Chaque patient ne s'en voit pas moins attribuer un lit particulier lors de son entrée dans une salle, attribution qui est rarement modifiée lors d'un séjour qui peut durer plusieurs mois. Le patient peut se retirer dans son lit où il dispose d'une couette sous laquelle il peut se «cacher». C'est à travers les résistances de ceux qui ne veulent pas sortir du lit malgré les changements d'optique thérapeutique appliqués dans les années 1960, que cette appropriation du lit devient visible pour l'historien à travers leur transcription dans les dossiers individuels.

\section{Porte}

Le problème de la porte du «lieu d'aisance», qui sert d'introduction à ce texte, suggère plusieurs pistes. L'échange entre Vellut et Vermeylen illustre les tensions qui caractérisent la psychiatrie de l'entre-deux-guerres. La fonction d'enfermement est toujours centrale: même si l'Institut dispose dès son inauguration de sections ouvertes, $80 \%$ de ses patients s'y trouvent sous le régime de la «collocation", c'est-à-dire d'une hospitalisation forcée. Certes les modalités de ces enfermements varient d'un pays à l'autre, mais les réformes d'ouverture sont plutôt rares dans l'Europe de l'entre-deux-guerres: l'enfermement est de loin le régime dominant jusqu'aux années 196034 . 
La psychiatrie de cette première moitié du XXe siècle recherche néanmoins, et avec constance, son inscription définitive dans le monde médical. Dans un premier temps, on envisage de construire à l'Institut des «w.c. [qui] sont en communication directe avec les dortoirs à raison de la surveillance permanente à laquelle sont soumis les malades»35. Mais un tel dispositif s'inscrit trop dans le paradigme sécuritaire et d'enfermement du XIX siècle que les décideurs politiques et psychiatres bruxellois tentent d'éviter à tout prix. Dans les discours destinés au grand public comme dans les échanges liés à la conception de l'Institut, transparaît la volonté de construire une institution médicale et non pas un asile, d'inscrire la psychiatrie dans le champ de la médecine, de faire preuve d'une certaine ouverture. Il faut dès lors suivre les recommandations de l'architecture hospitalière, et construire des «lieux d'aisance» individuels et fermés même si cela implique une surveillance moins totale (Depage, Vandervelde et Cheval 1912 : 175-176). D'où la minutie des instructions de Vermeylen à Vellut dans le document d'ouverture (document 1 ).

D’une manière générale, la porte, symbole de la fermeture, doit cloisonner définitivement un lieu. Dans la scène d'ouverture du film documentaire San Clemente de 1982, Raymond Depardon illustre l'hôpital psychiatrique par une grande porte qui demeure fermée pendant quelques dizaines de secondes avant qu'il ne puisse entrer dans l'hôpital. Or, le cloisonnement vers l'extérieur mais également à l'intérieur de l'hôpital pose problème par l'étanchéité complète qu'il crée, puisque le personnel doit pouvoir circuler, et parfois très rapidement en cas de problèmes rencontrés avec des patients agités. La possession de la clé était et reste jusqu'à aujourd'hui un élément distinctif entre patients et infirmières/médecins. Les infirmières, et plus particulièrement l'infirmièrevolante, sont responsables de la gestion des portes, et doivent chaque soir vérifier que les sorties vers l'extérieur sont bien closes et verrouillées ${ }^{36}$. Ce sont elles qui gèrent la circulation à l'intérieur de l'espace psychiatrique. Depuis les années 1950, des nouvelles portes sont installées dans les salles qui accueillent les patients les plus agités, des portes comportant des ouvertures vitrées afin de pouvoir surveiller en permanence les reclus. La possibilité technique d'utiliser à des frais jugés raisonnables du verre «securit» se brisant plus difficilement permet une transparence peu dangereuse ${ }^{37}$.

Le problème, pour l'ingénieur, est donc double: d'une part, il lui faut des portes et des serrures qui résistent à la force des patients et à des tentatives de fuite. Toutes les portes extérieures et intérieures sont dès lors munies de «serrures doubles, à gorges». Mais, d'autre part, le cloisonnement efficace des patients ne doit pas nuire à la libre circulation du personnel: il faut donc des serrures «absolument identiques entre-elles, de manière qu'avec une seule clef on puisse toutes les faire fonctionner» 38 . Les moyens techniques des années 1930 rendent ce compromis relativement difficile. Ainsi l'ingénieur souhaite avoir des serrures qui sont utilisables avec une clé générale, mais en même temps indivi- 
dualisées afin de pouvoir moduler au maximum l'accès à différents espaces psychiatriques. Or, aucune des entreprises de serrurerie de l'époque n'est capable de produire de telles serrures. L'écart est significatif entre la porte imaginée et la porte techniquement réalisable.

Du côté des patients, de nombreux dossiers contiennent des traces de cette «confrontation» avec la porte comme outil de réclusion. Mill M., un officier à la retraite d'une soixantaine d'années, trois fois interné contre son gré entre 1954 et 1958 pour «idées délirantes», apparaît particulièrement énervé par les portes. Son dossier est rempli de notes mentionnant des «attaques" répétées contre les portes: "Le malade est toujours très confus. Reste souvent devant la porte et la secoue pour sortir ${ }^{39}$ ». Dans le dossier de Solal M., un garçon de restaurant qui se retrouve en 1956 à l'Institut pour «éthylisme», l'infirmière note dès le premier jour: "veut se promener dans la salle, cherche une porte, ne veut pas rester ici 40 ».

Architectes et psychiatres d'hier, tout comme les historiens d'aujourd'hui, privilégient cette fonction de clôture. La porte n'est cependant pas seulement vécue comme un objet faisant barrière, mais aussi comme un objet qui protège. En effet, la porte fermée constitue également une protection de l'espace dans lequel on se trouve. Valérie L., internée en 1961 à cause de ses accès d'anxiété, se sent en relative sécurité à l'intérieur de «sa» salle. L'ouverture de la porte constitue pour elle un moment d'angoisse: «La malade semble craindre quelque chose car chaque fois qu'un médecin ouvre la porte elle sursaute et le regarde avec des yeux méfiants $4{ }$ ». Carole $S$. est une jeune femme de vingt-cinq ans qui atterrit à l'Institut à cause de ses «idées de suicide». Gérante d'une petite épicerie à Bruxelles, elle se sent rapidement à l'aise à l'Institut malgré le fait qu'elle soit soumise à une thérapie d'insuline et à des électrochocs: "Très déprimée, très anxieuse, regarde toujours la porte d'entrée croit qu'on vient la chercher pour Duffel 42 ». Pour elle, la porte est le lieu de la mauvaise nouvelle, à savoir l'annonce éventuelle d'un transfert vers un asile de province qui impliquerait à la fois un éloignement de sa famille et un diagnostic d'incurabilité.

Finalement, comme toute frontière, la porte est également un espace de rencontre, un espace d'entre-deux. Ainsi pour Robert C., qui écrit de nombreuses lettres à son médecin, se tenir près de «sa» porte, c'est dire qu'il est prêt à entrer en relation avec le médecin: "Je me tiens éventuellement à côté des portes, dans ma chambre-bureau, quand vous aurez le temps ${ }^{43}$ ».

\section{La trajectoire des objets}

Les exemples pourraient être multipliés: la fenêtre qui doit permettre la circulation de l'air mais non la circulation des «reclus» (voir document 8), le parc entourant l'Institut qui est pensé comme une barrière naturelle visant à empêcher les fuites, le hourdis spécifique qui est utilisé au premier étage du pavillon B 
- l'étage qui renferme les patients les plus remuants - afin de le rendre inaudible pour le reste de l'Institut, le système d'alarme mis en place pour appeler les infirmières en cas d'agitation non maîtrisable. Mais notre trio, qui couvre une gamme de formes et de fonctions, donne déjà accès à trois thématiques que la réflexion sur la vie sociale des objets permet de mettre en carte.

En premier, la thématique la plus évidente, mais qui n'a guère encore donné lieu à une réflexion approfondie par les sciences humaines, est l'interdépendance entre des objets qui déterminent la pratique psychiatrique quotidienne, et la psychiatrie qui transforme ces mêmes objets. Ainsi, le lit change de fonction par sa transposition d'une chambre ordinaire vers une salle d'un asile psychiatrique. Sur le plan symbolique, le lit qui procure du repos est transformé en outil de classification et d'enfermement. Au niveau matériel, ce changement se traduit par des adaptations légères mais significatives. À revers, le lit structure l'espace psychiatrique, formate l'agencement des salles.

Deuxièmement, l'expérience quotidienne de la psychiatrie par les médecins, le personnel soignant et les reclus n'a pas encore été pensée à travers la culture matérielle. Longtemps dominé par les psychiatres, le récit sur ce champ médical a été enrichi depuis une quinzaine d'années par la voix des patients ${ }^{44}$. Cette narration oublie encore un acteur qui est apparu dans les trois exemples: les infirmières ${ }^{45}$. Le cas de l'Institut est certes particulier: dès les années 1930, le personnel soignant de cette institution est entièrement composé de femmes infirmières diplômées, dont quelques-unes spécialisées en hygiène mentale. Dans la plupart des asiles en Belgique et en Europe, le "personnel soignant» - terminologie anachronique - est souvent composé de personnes sans formation spécifique. Leur fonction est avant tout celle de surveillance, et leur appellation de «gardien» qui indique aussi que les hommes, contrairement à d'autres services hospitaliers, y sont beaucoup plus représentés. Cette population infirmière apparaît particulièrement centrale dans la vie quotidienne de l'institution et dans la pratique des différents dispositifs. Ce sont d'ailleurs dans les manuels qui s'adressent plus particulièrement à cette catégorie professionnelle qu'on trouve des indications précises sur la matérialité de ces objets et sur leur utilisation, les précis de psychiatrie s'avérant beaucoup plus silencieux sur ces objets qui structurent le quotidien psychiatrique. Comprendre l'institution psychiatrique nécessite une plus grande attention à ce corps intermédiaire qui détermine la transformation d'objets pensés (bâtiments, lits...) en objets agis.

Finalement, penser ces éléments en termes de «biographie» (Kopytoff 1986) permet de dépasser une description statique de ces objets qui connaissent au moins une triple vie. Dans un premier temps, ils sont pensés et, dans le cas de l'Institut, par un ingénieur-architecte et un psychiatre dont les cadres de références sont multiples. Vellut a déjà participé à la construction de plusieurs hôpitaux, et les deux hommes visitent à partir de la fin des années 1920 d'autres asiles en Belgique et à l'étranger, mais également des prisons. À ce moment, l'asile est encore imaginé 


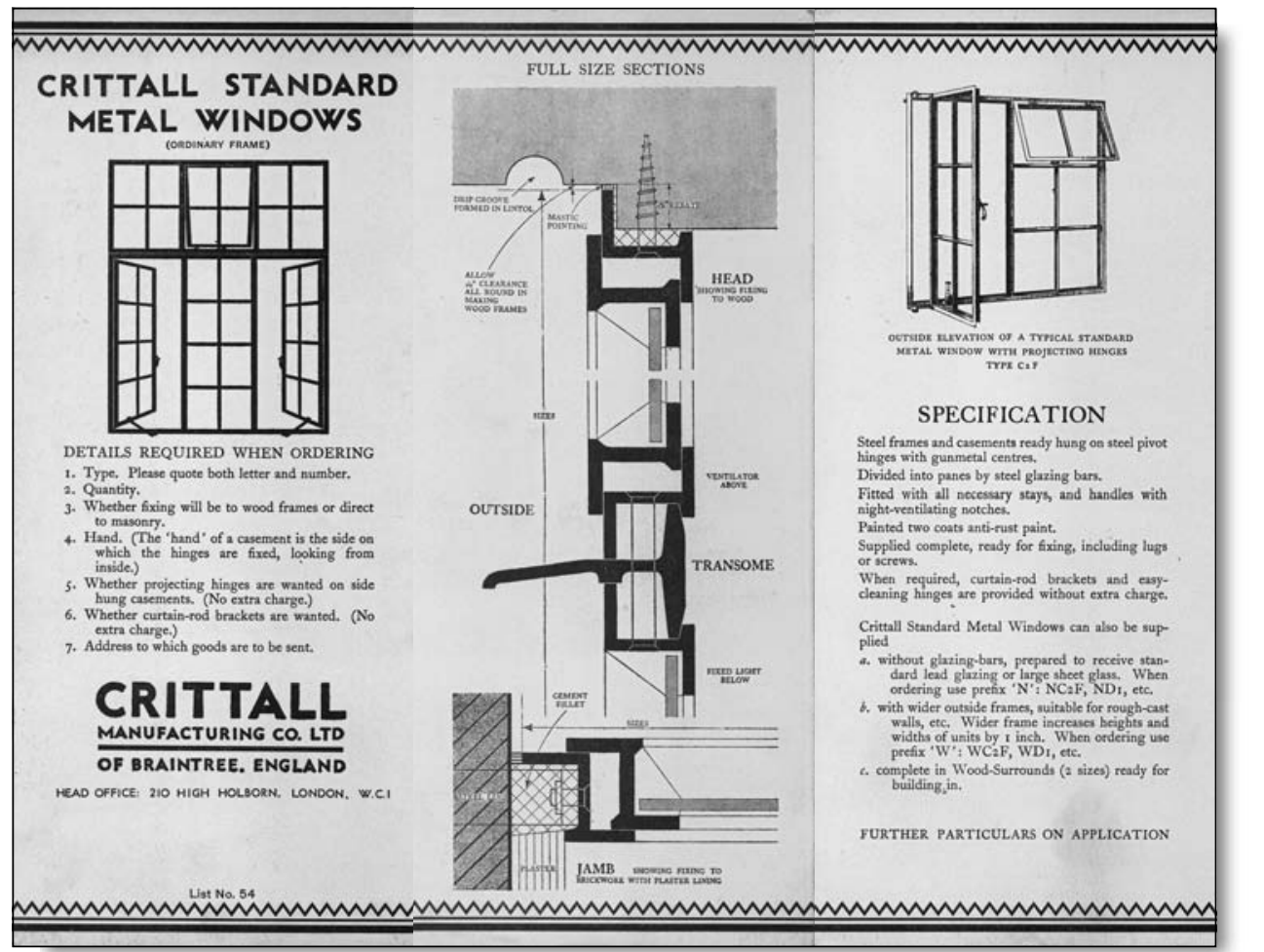

Document 8. Publicité pour les cadres de fenêtres (début des années 1930).

(C) Crittall Manufacturing Compagny LTD

comme un espace clos, comme un espace d'enfermement. Mais simultanément, la vague antipsychiatrique qui secoue l'Europe depuis la fin du XIXe siècle laisse des traces sur les objets meubles et immeubles qui peuplent l'Institut: les effets négatifs que provoque l'impression du cloisonnement sont pris en compte (Schmiedebach 1996; Fauvel 2006). Il s'agit de créer au niveau architectural l'impression de l'ouverture: le grillage du jardin disparaît derrière des haies, le cadre en acier des fenêtres est caché derrière un bois de placage. Dans un deuxième temps, ces objets imaginés doivent être transformés en objets existants. Cette transformation se heurte à de nombreux obstacles - impossibilité technique, fabrication défaillante... - mais la discussion entre architectes/psychiatres et entrepreneurs s'avère également productive car elle intègre un nouvel espace d'expérience. L'artisan, dans sa réponse au cahier de charges, propose souvent des solutions auxquelles ni l'ingénieur ni le psychiatre n'avaient pensé. Puis, c'est seulement dans une troisième étape que ces objets deviennent des «objets agis», des objets qui sont incorporés par des acteurs. Chacune de ces vies, et leur interaction, appelle notre attention. Ce qui est particulièrement intéressant est l'écart qui peut exister entre l'objet imaginé et l'objet agi. D'autant que, à côté de la fonction d'objet agi, la baignoire, la porte et le lit devien- 
nent aussi des «objets agissants» qui structurent et transforment la pratique et l'expérience psychiatrique. En partant de la culture matérielle des asiles, l'historienne australienne Catharine Coleborne parle du mythe de l'hôpital psychiatrique qui ne serait que rarement tel que les gens l'auraient imaginé (2003: 99). C'est en interrogeant ces trois vies, pour elles-mêmes et dans leur interaction, que nous pouvons travailler sur cette distance et ce décalage, dont les chercheurs en sciences humaines et sociales sont peut-être tout autant victimes que «les gens».

\section{Ouvrages cités}

ANKELE, Monika. 2009. Alltag und Aneignung in Psychiatrien um 1900.

Selbstzeugnisse von Frauen aus der Sammlung Prinzhorn. Vienne, Böhlau.

ApPadurai, Arjun (éd.). 1986. The Social Life of Things. Commodities in Cultural Perspective. Cambridge, Cambridge University Press.

ATTFIELD, Judy. 2000. Wild Things. The Material Culture of Everyday Life. Oxford, Berg (Materializing Culture).

BARReTt, Robert J. 1996. The Psychiatric Team and the Social Definition of Schizophrenia. An Anthropological Study of Person and Illness. New York, Cambridge University Press. (Studies in Social and Community Psychiatry).
BorcK, Cornelius. 2005. Hirnströme. Eine Kulturgeschichte der Elektroenzephalographie.

Göttingen, Wallstein

(Wissenschaftsgeschichte).

Boschma, Geertje. 2003. The Rise of Mental Health Nursing. A History of Psychiatric Care in Dutch Asylums, 1890-1920. Amsterdam, Amsterdam University Press.

BRASLOW, Joel T. 1997. Mental Ills and Bodily Cures. Psychiatric Treatment in the First Half of the Twentieth Century. Berkeley, University of California Press (Medicine and Society).

Clarke, Liam. 1993. «The Opening of Doors in British Mental Hospitals in the 1950s", History of Psychiatry, vol. 4, n ${ }^{\circ}$ 16: 527-551. 
Coleborne, Catharine. 2003.

«Remembering Psychiatry's Past:

The Psychiatric Collection and its Display at Porirua Hospital Museum, New Zeland», Journal of Material Culture, vol. 8, n 1: 97-118.

Depage, Antoine, Paul Vandervelde et Victor Cheval. 1912 [1907-1909]. La construction des hôpitaux. Étude critique. Bruxelles, Misch et Thron.

Fauvel, Aude. 2006. «Violence aliéniste, l'asile de Clermont (Oise). Mythe et réalité», in Anne-Claude Ambroise-Rendu, Fabrice d'Almeida et Nicole Edelman (éd.), Des gestes en histoire. Formes et significations des gestualités médicale, guerrière et politique. Paris, Seli Arslan (Histoire, cultures et sociétés) : 53-67.

Foucault, Michel. 1975. Surveiller et punir. Naissance de la prison. Paris, Gallimard (Bibliothèque des histoires).

Goffman, Erving. 1968 [1961]. Asiles. Études sur la condition sociale des malades mentaux et autres reclus. Paris, Minuit (Le sens commun), (éd. orig., Asylums. Essays on the Social Situation of Mental Patients and Other Inmates. Garden City [NY], Anchor Books Doubleday).

Grob, Gerald N. 1991. From Asylum to Community. Mental Health Policy in Modern America. Princeton, Princeton University Press.

HenCKes, Nicolas. 2007. «Le nouveau monde de la psychiatrie française. Les psychiatres, l'État et la réforme des hôpitaux psychiatriques de l'après-guerre aux années $1970 »$, thèse de doctorat de sciences sociales, École des hautes études en sciences sociales, Paris.

Keller, Richard C. 2007. Colonial Madness. Psychiatry in French North Africa.

Chicago, University of Chicago Press.

KopyTOFF, Igor. 1986. «The Cultural Biography of Things: Commoditization as Process ", in Arjun Appadurai (éd.) : 64-91.

LAÉ, Jean-François. 2009. «Le lit, dispositif de l'institution totale", in Philippe Artières et Jean-François Laé (éd.), L'asile aux fous. Un lieu d'oubli. Saint-Denis, Presses universitaires de Vincennes: 99-117.
LANDZELIUS, Kyra Marie. 2001. «Charged

Artifacts and the Detonation of Liminality:

Teddy-Bear Diplomacy in the Newborn

Incubator Machine», Journal of Material Culture, vol. 6, n 3: 323-344.

LATOur, Bruno. 1987. Science in Action. How to Follow Scientists and Engineers through Society. Cambridge (Mass.), Harvard University Press.

LECLERCQ, Valérie. 2009. «Le stylo-bille et l'entonnoir. Écrits et écriture des patients de l'Institut de psychiatrie Brugmann (1931-1980)», mémoire de master, Université libre de Bruxelles.

LÜDTKE, Alf. 1994. «Eigensinn», in Berliner Geschichtswerkstatt (éd.), Alltagskultur, Subjektivität und Geschichte. Zur Theorie und Praxis von Alltagsgeschichte. Münster, Westfälisches Dampfboot: 139-153.

Majerus, Benoît. 2010. «Une stabilisation difficile. La chlorpromazine dans les années 1950 en Belgique», Gesnerus. Swiss Journal of the History of Medicine and Sciences, vol. $67, \mathrm{n}^{\circ} 1: 57-72$.

Mignot, Roger et Ludovic Marchand. 1931 [1912]. Manuel technique de l'infirmier des établissements d'aliénés, à l'usage des candidats aux diplômes d'infirmier des asiles. Paris, Gaston Doin.

Morgenthaler, Walter et Oscar-Louis FOREL. 1940 [1930]. Manuel de psychiatrie à l'usage du personnel infirmier. Berne, Hans Huber (éd. orig., Die Pflege der Gemüts-und Geisteskranken).

NolTE, Karen. 2003. Gelebte Hysterie: Erfahrung, Eigensinn und psychiatrische Diskurse im Anstaltsalltag um 1900.

Francfort-sur-le-Main, Campus

(Geschichte und Geschlechter). - 2009. «Vom Verschwinden der Laienperspektive aus der Krankengeschichte: Medizinische Fallberichte im 19. Jahrhundert», in Sibylle Brändli, Barbara Lüthi et Gregor Spuhler (éd.), Zum Fall machen, zum Fall werden. Wissensproduktion und Patientenerfahrung in Medizin und Psychiatrie des 19. und 20. Jahrbunderts.

Francfort-sur-le-Main, Campus: 33-61. 
OOSTERHUIS, Harry. 2005. «Insanity and Other Discomforts. A Century of Outpatient Psychiatry and Mental Health Care in the Netherlands 1900-2000", in Marijke Gijswijt-Hofstra et al. (éd.), Psychiatric Cultures Compared. Psychiatry and Mental Health Care in the Twentieth Century: Comparisons and Approaches. Amsterdam, Amsterdam University Press: 73-102.

ParrotT, Fiona R. 2005. «It's Not Forever": The Material Culture of Hope», Journal of Material Culture, vol. 10, n ${ }^{\circ} 3$ : 245-262.

QuÉTEL, Claude. 2009. Histoire de la folie. De l'Antiquité à nos jours. Paris, Tallandier.

Reaume, Geoffrey. 2000. Remembrance of Patients Past. Patient Life at the Toronto Hospital for the Insane, 1870-1940.

New York, Oxford University Press.

RÉGIS, Emmanuel. 1923 [1885]. Précis de psychiatrie. Paris, Octave Doin (1re éd., [sous le titre] Manuel pratique de médecine mentale).
SCHMIEDEBACH, Heinz-Peter. 1996. "Eine "antipsychiatrische Bewegung" um die Jahrhundertwende», in Martin Dinges (éd.), Medizinkritische Berwegungen im Deutschen Reich (ca. 1870-ca. 1933). Stuttgart, Franz Steiner (Medizin, Gesellschaft und Geschichte): 127-159.

Thomas, Gregory M. 2004. «Open Psychiatric Services in Interwar France», History of Psychiatry, vol. 15, n 2: 131-153.

Topp, Leslie, James E. MorAN et Jonathan Andrews (éd.). 2007. Madness, Architecture and the Built Environment. Psychiatric Spaces in Historical Context. Londres, Routledge (Routledge Studies in the Social History of Medicine).

VijSELAAR, Joost. 2010. Het gesticht. Enkele reis of retour. Amsterdam, Uitgeverij Boom.

YANNI, Carla. 2007. The Architecture of Madness. Insane Asylums in the United States. Minneapolis, University of Minnesota Press (Architecture, Landscape, and American Culture).

\section{Notes}

* Je remercie Bruno Benvindo et Kenneth Bertrams, ainsi que les lecteurs anonymes de la revue pour leurs corrections et leurs commentaires.

1. Georges Vellut (1866-1961), ingénieur de formation, construit plusieurs bâtiments hospitaliers dans la région bruxelloise pour la CAP de Bruxelles qui est son employeur depuis 1902.

2. Pour faciliter la lecture, j'emploierai désormais le terme «Institut» avec une majuscule pour désigner l'Institut de psychiatrie de l'hôpital Brugmann à Bruxelles.

3. Sans avoir directement servi de références pour cette contribution, les travaux d'Arjun Appadurai (1986) et Bruno Latour (1987) constituent un des arrière-fonds de la réflexion.

4. Voir encore récemment l'attaque contre le récit foucauldien menée par Claude Quétel en défense des psychiatres (2009).
5. ACPASB, travaux, boîte 52, lettre du directeur délégué de la $2^{\mathrm{e}}$ section de la $2^{\mathrm{e}}$ direction générale du ministère de la Justice au président de la CAP (non datée, probablement seconde moitié des années 1930).

6. ACPASB, AGP [Affaires générales et programmation]-CAP, boîte 159 , lettre du 1er octobre 1936 de Vermeylen à la CAP.

7. ACPASB, Madeleine, «De la psychiatrie», 1992.

8. Hôpital Brugmann, Institut de psychiatrie (par la suite HBIP), Ancienne Série (AS), n 12315, lettre du psychiatre au juge des enfants (6 décembre 1956). Le nom des patients a été anonymé.

9. Le patient est mis en camisole de force qui est souvent en grosse toile.

10. HBIP, AS, $\mathrm{n}^{\circ} 12315$, note des infirmières (13 novembre 1956). L'orthographe des notes a été maintenue. 
11. Ibid., n $^{\circ} 7666$, notes du 4 et 5 octobre 1939 .

12. Ibid., $\mathrm{n}^{\circ} 5103$, note des infirmières (12 décembre 1936).

13. Ibid., $\mathrm{n}^{\circ} 9885$, note des infirmières (3 février 1960).

14. Ibid., n’ 3360, note des infirmières (23 mai 1940).

15. Ibid., n ${ }^{\circ} 3930$, note des infirmières (20 février 1940). Dans une autre notice du 11 novembre 1939, on retrouve cette écriture "pudique»: "Présente une petite ecchymose sur le bras gauche. [...] Son mari dit-elle n'a pas beaucoup de patience avec elle».

16. «Mabouls Palaces - chez les fous», Le Pétard, 8 août 1936.

17. HBIP, AS, n 1348, Lettre de James B. à sa femme, 10 octobre 1933.

18. Ibid., Lettre de James B. à sa femme, 7/09/1933.

19. Ibid., n 10290, note médicale, 30 octobre 1953.

20. Ibid., note des infirmières, 1er janvier 1955.

21. Dans les années 1990, l'eau est réintroduite dans l'arsenal thérapeutique de la psychiatrie, pour ses effets relaxant et apaisant.

22. ACPASB, travaux, boîte 30 , lettre de Dom à Vellut (16 novembre 1929).

23. L'asile de Lovenjoel est un des plus grands asiles psychiatriques de Belgique, institution gérée par une congrégation religieuse. Il sert en même temps d'hôpital universitaire à l'Université catholique de Louvain.

24. ACPASB, travaux, boîte 30, lettre de la Mère Révérende de Lovenjoel à Georges Vellut (23 novembre 1929).

25. Ibid., travaux, boîte 31b, lettre de Dom à Vellut (16 novembre 1929).

26. Ibid., travaux, boîte 30 , relevé détaillé des objets fournis par l'intermédiaire du service des Travaux pour l'équipement de l'Institut de psychiatrie (non daté). Il y a trente-six planches pour cent seize lits, et les planches ne sont pas installées de manière systématique pour adoucir les aspects « rébarbatifs».

27. Ibid., travaux, boîte 219 , cahier des charges concernant les planches pour le lit (non daté, vers 1930).

28. Ibid., travaux, boite 30 , dossier concernant la fourniture de 144 armoires-vestiaires en fer pour l'Institut de psychiatrie.

29. Paul Danblon, «Les maladies mentales», Le point de la médecine, Bruxelles, RTB (Radio télévision belge), 21 décembre 1964.
30. HBIP, AS, $n^{\circ} 4530$, note des infirmières, 17 juillet 1956.

31. Ibid., n 1230 , note des infirmières, 4 janvier 1933.

32. HBIP, Nouvelle Série (NS), $n^{\circ} 18000$, fiche des infirmières, 16 mars 1978.

33. Les années 1950 et 1960 sont souvent considérées comme un tournant dans l'historiographie de la psychiatrie (Vijselaar 2010 : 337-341). Pour la justifier, les auteurs mettent en avant la sortie de la psychiatrie des structures asilaires (Grob 1991; Henckes 2007) et l'apparente rupture thérapeutique introduite par les neuroleptiques, rupture qui est cependant à interroger (Majerus 2010).

34. Pour la France voir Gregory M. Thomas (2004) et Richard C. Keller (2007), pour les PaysBas, Harry Oosterhuis (2005) et pour l'Angleterre Liam Clarke (1993).

35. ACPASB, travaux, boîte 30 , séance du Conseil supérieur d'hygiène (31 mars 1928).

36. ACPASB, fonds Capon, longuette (inscription du 27 avril 1941). La longuette est le règlement officieux des infirmières à l'Institut. Chaque nouvelle infirmière passe les deux premiers jours à lire ce cahier manuscrit avant de pouvoir accéder à la salle qui lui a été attribuée.

37. ACPASB, travaux, boîte 219 , échange de lettres entre le directeur de l'hôpital Brugmann (hôpital dont dépend l'Institut de psychiatrie) et le conseil d'administration de la CAP.

38. Ibid., travaux, boîte 30 , cahier des charges pour l'Institut de psychiatrie.

39. HBIP, AS, $n^{\circ} 10455$, note des infirmières (8 novembre 1957).

40. Ibid., n 11910, note des infirmières (4/07/1956).

41. Ibid., $\mathrm{n}^{\circ} 14175$, note des infirmières (13 septembre 1961).

42. Ibid., n 9630, note des infirmières (28/07/1952).

43. Lettre de Robert C. à la médecin-adjoint (25 janvier 1961), cité d'après Valérie Leclercq (2009: 34).

44. Voir entre autres Reaume (2000), Nolte (2003), Ankele (2009) et Vijselaar (2010).

45. Boschma (2003) fait figure d'exception. 